Pacific Journal of Mathematics

HALF RINGS IN LINEAR SPACES 


\title{
HALF RINGS IN LINEAR SPACES
}

\author{
P. H. MASERICK
}

Von Neumann and Zaanen have studied measure theoretic properties of collections of sets which satisfy weaker axioms than those of a ring. In this paper it is shown that the von Neumann axioms for a half ring of sets and the Zaanen axioms for a semi-ring of sets can be weakened without loss of their measure theoretic significance.

An investigation of the geometrical structure of a collection $\mathscr{R}$ of convex sets which satisfy either von Neumann's, Zaanen's or our weaker axioms is conducted. Principally we extend some earlier results by showing that under rather mild restrictions, sets of such collections are polyhedral. After imposing the additional condition that $\mathscr{R} \backslash\{\phi\}$ be a neighborhood base for a linear topology, we prove that if $\mathscr{R}$ is a semi-ring in the earlier sense then the topology induced by $\mathscr{R}$ is a so called weak topology and conversely every weak topology has such a neighborhood base. Finally we characterize subspaces of the Banach space $\left(c_{0}\right)$ as the only Banach spaces which have a neighborhood base of convex sets which together with the null set form a half ring (in the weaker sense).

Our set theoretic notations, definitions and conventions are standard. The reader might observe, however, that the word countable as used in this paper, refers to finite sets (including the null set) as well as to those infinite sets with the cardinality of the integers. In $\S 1$ through $\S 4$ our terminology, for the most part, adheres with that established in [1]. The scalar field of all linear spaces $X$ considered will be assumed to be the real numbers. If $x, y \in X$, then the expression $(x, y),(x, y]$ or $[x, y]$ will denote either the open, half open or closed line segment whose endpoints are $x$ and $y$. All linear topological spaces $X$ considered will be assumed to satisfy the Hausdorff separation axiom. If $A \subset X$, then the symbols $\mathrm{cl}(A)$ and $\operatorname{int}(A)$ will denote the topological closure and interior of $A$ respectively. A subset $A$ will be called nondegenerate if $\operatorname{int}(A) \neq \varnothing$. The notation $\left(c_{0}\right)$ will be used to designate the Banach space of all real valued sequences $x=\left(x_{1}, x_{2}, \cdots,\right)$ which converge to zero and are normed by $\|x\|=\sup _{i}\left|x_{i}\right|$. If $X$ is a linear topological space and $\Gamma$ is its set of continuous linear functionals (i.e., the topological) dual of $X$ ) then the weak topology of $X$ will refer to the coarsest linear topology in which every member of $\Gamma$ is continuous. It should be recalled that the collection of all sets of the form

Received July 25, 1961. 


$$
\bigcap_{i=1}^{k}\left\{x \in X|| f_{i}(x) \mid \leqq \varepsilon\right\}
$$

where $f_{i} \in \Gamma$ and $\varepsilon>0$ is an equivalent neighborhood base at the origin for the weak topology. The undefined measure theoretic terms which appear in $\S 5$ agree with [2].

2. Demi-spaces, polyhedra and polytopes. In a conversation with the author, Professor P. C. Hammer defined a convex subset of a linear space to be a demi-space if its complement is also convex. Algebraically open and closed half spaces are examples of demi-spaces. The main properties of demi-spaces which we will be using can all be derived from Theorem 2.1 below and its corollary. The theorem is due to Kakutani and a proof appears in [3, Th. 1.8].

THEOREM 2.1. (Kakutani). If $P, Q$ are disjoint convex subsets of a linear space, then there exists a demi-space $D$ such that $P \subset D$ and $Q \subset X \backslash D$. i.e., The demi-space $D$ strictly separates $P$ from $Q$.

Corollary 2.2. A subset $D$ of a linear space $X$ is a demispace if and only if it is a maximal convex set which excludes some convex set.

If one defines a semi-space [3] as a maximal convex set which excludes a point, then Corollary 2.2 implies that these sets are also examples of demi-spaces.

In [7], Weyl defines a convex polyhedron as a subset of a linear space which can be realized as the intersection of finitely many half spaces. Here we use a more general definition and define a convex polyhedron as a subset of a linear space which can be expressed as the intersection of a finite number of demi-spaces.

In [4] we generalized Weyl's definition of a convex polyhedron to embrace certain bounded convex sets in infinite dimensional spaces. We showed that this definition was well motivated and shared many of the properties of the former. In fact the only bounded nondegenerate convex polytopes which exist in finite dimensional normed linear spaces were seen to be polyhedra. The definition given there is as follows: If $\left\{D_{\alpha} \mid \alpha \in A\right\}$ is a collection of algebraically closed half space in a linear space $X$ such that

(i) $P=\cap\left\{D_{\alpha} \mid \alpha \in A\right\}$.

(ii) For each $x \in X$ there exists $\alpha_{1}, \alpha_{2}, \cdots, \alpha_{n} \in A$ having the property that $x \in \cap\left\{D_{\alpha} \mid \alpha \in A, \alpha \neq \alpha_{1}, \alpha_{2}, \cdots, \alpha_{n}\right\}$, then $P$ is a convex polytope.

For the purpose of this paper we will broaden this definition by 
allowing the collection $\left\{D_{\alpha} \mid \alpha \in A\right\}$ to be a collection of demi-spaces. Every collection of demi-spaces which satisfies (i) and (ii) will be called a representative collection of demi-spaces for $P$. Theorem 2.3 links our definition of a convex polytope to that given in [4] and enables us to more fully utilize the results of [4].

THEOREM 2.3. If $P$ is a nondegenerated convex polytope in a linear topological space $X$ and if $\left\{D_{\alpha} \mid \alpha \in A\right\}$ is a representative collection of demi-spaces for $P$, then $\mathrm{cl}(P)$ is a convex polytope in the sense of [4] as well as in our sense and $\left\{\mathrm{cl}\left(D_{\alpha}\right)\right\}$ is a representative class of half spaces for $\mathrm{cl}(P)$.

Proof. Since $P$ has a nonvoid interior so does each demi-space $D_{\alpha}$. Thus it is well know [1] that there is a continuous linear functional $f_{\alpha}$ and a real number $c_{\alpha}$ such that

(a) $x \in D_{\alpha}$ implies $f_{\alpha}(x) \leqq c_{\alpha}$

(b) $x \in D_{\alpha}$ implies $f_{\alpha}(x) \geqq c_{\alpha}$.

Let $E_{\alpha}=\left\{x \in X \mid f_{\alpha}(x) \leqq c_{\alpha}\right\}$. Then (a) implies $P \subset \bigcap_{\alpha} E_{\alpha}$, so that $\operatorname{cl}(P) \subset \bigcap_{\alpha} E_{\alpha}$. Suppose $p \in \bigcap_{\alpha} E_{\alpha}$ and let $y \in \operatorname{int}(P)$. Then $f_{\alpha}(y)<c_{\alpha}$ for each $\alpha \in A$. Thus $f_{\alpha}(z)<c_{\alpha}$ for each $z$ in the open line segment $(p, y)$. But then (b) implies $z \in \bigcap_{\alpha} D_{\alpha}=P$ so that $P \in \operatorname{cl}(P)$. Therefore $\operatorname{cl}(P)=\bigcap_{\alpha} E_{\alpha}$. Finally since $\bigcap_{\alpha} D_{\alpha}$ is a convex polytope and since $E_{\alpha} \supset D_{\alpha}$, it is clear that $\left\{E_{\alpha} \mid \alpha \in A\right\}$ satisfies (ii) above.

If we define a face of a convex subset of a linear topological space to be a maximal convex subset of its boundary, then Theorem 2.3 and the results of [4] imply:

Corollary 2.4. A nondegenerate convex polytope in a linear topological space has a countable number of faces if and only if it has a countable representative class of demi-spaces.

3. Half rings, polyhedra and polytopes, A collection $\mathscr{R}$ of sets along with the empty set will be called a half ring if

(i) For each $R_{1}, R_{2} \in \mathscr{R}$ the intersection $R_{1} \cap R_{2} \in \mathscr{R}$.

(ii) For each $R, R^{\prime} \in \mathscr{R}$ such that $R \subset R^{\prime}$ there exists a countable chain $\left\{R_{i}\right\} \subset \mathscr{R}$ such that $R=R_{1} \subset R_{2} \subset \cdots \subset \bigcup_{i} R_{i}=R^{\prime}$ and $R_{i} \backslash R_{i-1} \in \mathscr{R}$ for each $i>1$.

If the ascending chain of (ii) can always be chosen to be finite then $\mathscr{R}$ will be called a half ring which satisfies the finite chain condition. The latter type half ring was defined by von Neumann [6] who investigated their measure theoretic importance. Note that (ii) implies:

(iii) For each pair $R, R^{\prime} \in \mathscr{R}$ such that $R \subset R^{\prime}$, there exists a countable sequence $\left\{P_{i}\right\}$ of pairwise disjoint members of $\mathscr{R}$ such that $R=P_{1}$ and $R^{\prime}=\bigcup_{i} P_{i}$.

A collection of sets which satisfy (i) and (iii) will be called a 
semi-ring. If a finite sequence can always be found for each such pair $R$ and $R^{\prime}$ then $\mathscr{R}$ will be called a semi-ring which satisfies the finite sequence condition. Zaanen [8] investigated the measure theoretic properties of the latter type semi-ring. In $\S 5$ we will extend these results to an arbitrary semi-ring. In this section and the next, however, we will extend the research presented in [5] by investigating the geometric properties of semi-rings and half rings of convex sets.

Theorem 3.1. If $\mathscr{D}$ is a collection of demi-spaces which is closed under complementation, then the collection $\mathscr{R}$ of all convex polytopes (polyhedra) which has a countable (finite) subset of $\mathscr{D}$ as a representative class is a half ring (half ring which satisfies the finite chain condition). If moreover $\mathscr{D}$ is translation invariant, so is $\mathscr{R}$.

Proof. We will prove the assertion only for the case where $\mathscr{R}$ is a collection of convex polytopes and leave the other to the reader. Since (i) of the half ring definition is obviously satisfied by $\mathscr{R}$ we need only show that $\mathscr{R}$ satisfies (ii). Suppose $R, R^{\prime} \in \mathscr{R}$ such that $R \subset R^{\prime}$, and let $\left\{D_{i}\right\}$ be a countable subcollection of $\mathscr{D}$ which is a representative class of demi-spaces for $R$. For each integer $k$ let

$$
R_{k}=\left(\bigcap_{i \geqq k} D_{i}\right) \cap R^{\prime} \text {. }
$$

Clearly $R_{k} \in \mathscr{R}$ for each integer $k$ and from (ii) of the definition of a convex polytope it follows that $\mathrm{U}_{k} R_{k}=R^{\prime}$. Moreover since

$$
\begin{aligned}
R_{k} \backslash R_{k-1} & =R^{\prime} \cap\left(\bigcap_{i \geqq k} D_{i} \backslash \bigcap_{i \geqq k-1} D_{i}\right) \\
& =R^{\prime} \cap\left(\bigcap_{i \geqq k} D_{i}\right) \cap\left(X \backslash D_{k-1}\right)
\end{aligned}
$$

we see that this set is a member of $\mathscr{R}$ for each $k>1$.

If we define a collection of subsets of space $X$ to be entire whenever it contains $X$ as one of its members, then Theorems 3.2 and 3.3 are partial converses to Theorem 3.1.

THEOREM 3.2. If $\mathscr{R}$ is an entire half ring of conver sets, then each $R \in \mathscr{R}$ is a convex polytope which has a countable representative class of demi-spaces.

Proof. Let $R$ be an arbitrary member of $\mathscr{R}$ and $\left\{R_{i}\right\}$ be the countable chain from $R$ to $X$ referred to in (ii) of the definition of a half ring. Then for each integer $i$ there is a demi-space $D_{i}$ which contains $R_{i}$ but strictly separates $R_{i}$ from $R_{i+1} \mid R_{i}$ (Theorem 2.1). 
Clearly $R=\bigcap_{i} D_{i}$ and if $x \in R$ then there exists $j$ such that $x \in R_{j+1} \backslash R_{j}$. Thus $x \in \cap\left\{D_{i} \mid i \geqq j+1\right\}$ so that $R$ is seen to be a convex polytope with $\left\{D_{i}\right\}$ as its representative class of demi-spaces.

It is not true that every member of an entire semi-ring of convex sets is a convex polytope. However we may state:

THEOREM 3.3. If $\mathscr{R}$ is an entire semi-ring of convex sets which satisfies the finite sequence condition (and hence if $\mathscr{R}$ is an entire half ring which satisfies the finite chain condition) then each $R \in \mathscr{R}$ is a convex polyhedron.

Proof. If $R$ is an arbitrary member of $\mathscr{R}$ and $\left\{R_{i} \mid i=1,2, \cdots, k\right\}$ is the partition of $X$ assured by (iii) of the definition, then from Theorem 2.1, for each integer there exists a demi-space $D_{i}$ which contains $R$ and strictly separates $R$ from $R_{i}$. It then follows that $R=\bigcap_{i} D_{i}$.

CoRollary 3.4. If $\mathscr{R}$ is an entire semi-ring of convex sets, then each $R \in \mathscr{R}$ can be expressed as the intersection of a countable number of demi-spaces.

4. Half rings and linear topologies. In the first part of this section we characterize the weak topology as the only linear topology which has a half ring $\mathscr{R}$ (of convex polyhedra), which satisfies the finite chain condition such that $\mathscr{R} \backslash\{\varnothing\}$ is a neighborhood base. In the latter part of the section, subspaces of the Banach space $\left(c_{0}\right)$ are similarly characterized in terms of half rings of convex polytopes. As a preliminary result we begin with:

THEOREM 4.1. If $\mathscr{P}$ is a neighborhood base of convex polyhedra for a locally convex topology, then that topology is equivalent to its weak topology.

Proof. By definition the given topology is at least as strong as the weak topology. Let $P \in \mathscr{P}$ be an arbitrary neighborhood of the origin. To see that the topologies are equivalent we need only prove the existence of a weak neighborhood of the origin which is contained in $P$. For this, let $\left\{D_{i} \mid i=1,2, \cdots, k\right\}$ be a finite collection of demispaces whose intersection is $P$. Since each $D_{i}$ has a nonvoid interior, as is well known [1], it can be separated from its convex complement by a continuous linear functional. Thus for each integer $i$ there is a continuous linear functional $f_{i}$ such that $X \backslash D_{i} \subset\left\{x \in X \mid f_{i}(x) \geqq c_{i}\right\}$, so that $P=\bigcap_{i} D_{i} \supset \bigcap_{i}\left\{x \in X \mid f_{i}(x)<c_{i}\right\}$ and the set on the right side of the contains symbol is the desired neighborhood. 
THEOREM 4.2. If $\mathscr{R}$ is a semi-ring of convex sets which satisfies the finite sequence condition such that $\mathscr{R} \backslash\{\varnothing\}$ is a neighborhood base for a linear topology on a linear space $X$, then the subcollection $\mathscr{R}_{0}$ of all convex polyhedra in $\mathscr{R}$ is a coarser semi-ring which satisfies the finite sequence condition, and such that $\mathscr{R}_{0} \mid\{\varnothing\}$ is an equivalent neighborhood base. If, moreover, $\mathscr{R}$ is a half ring which satisfies the finite chain condition then $\mathscr{R}_{0}$ is also a half ring which satisfies the finite chain condition. In either event, however, the given topology is a weak topology.

Proof. The last assertion is an immediate censequence of the first and Theorem 4.1. To establish the first assertion let $R \in \mathscr{R}$ be an arbitrary neighborhood of $p \in X$. Since $X$ is a linear topological space it is regular. Thus there exists $R_{1} \in \mathscr{R}$ such that $p \in \operatorname{int}\left(R_{1}\right)$ and $\operatorname{cl}\left(R_{1}\right) \subset \operatorname{int}(R)$. To see that $R_{1}$ is a convex polyhedron let $\left\{R_{i} \mid i=1,2, \cdots, k\right\}$ be the finite sequence of $R$ guaranteed by (iii) of the definition of a semi-ring. From Theorem 2.1, for each integer $i>1$, there exists a demi-space $D_{i}$ such that $D_{i} \supset R_{1}$ and $X \backslash D_{i} \supset R_{i}$. If $x \in R_{1}$ then the half open line segment $(p, x]$ contains a point $y$ (possibly $x$ itself) such that $y \in R \backslash R_{1}$. Thus $y \in R_{i}$ for some integer $i \geqq 2$ so that $y \notin D_{i}$. Since $p \in D_{i}$ and $D_{i}$ is convex it follows that $x \notin D_{i}$ so that $R_{1}=\bigcap_{i} D_{i}$. Thus if $R \in \mathscr{R}$ is a neighborhood of $p \in X$ there is a convex polyhedron in $\mathscr{R}$ which is contained in $R$ and is also a neighborhood of the point $p$. Therefore the collection $\mathscr{R}_{0}$ of all nondegenerate convex polyhedra in $\mathscr{R}$ is an equivalent neighborhood base.

To see that $\mathscr{R}_{0}$ is a semi-ring which satisfies the finite sequence condition, let $R, R^{\prime} \in \mathscr{R}_{0}$ such that $R \subset R^{\prime}$. Suppose further that $\left\{R_{i} \mid i=1,2, \cdots, k: R_{1}=R\right\}$ is a finite subcollection of pairwise disjoint members of $\mathscr{R}$ whose union is $R^{\prime}$ and let $i$ be arbitrarily chosen. We need only show that $R_{i}$ is a convex polyhedron. For each integer $n(n \neq i, n=1,2, \cdots, k)$ there exists, by Theorem 2.1, a demi-space $D_{n}$ such that $D_{n} \supset R_{i}$ and $X \backslash D_{n} \supset R_{n}$. Thus

$$
R^{\prime} \cap\left[\bigcap_{n \neq i} D_{n}\right] \supset R_{i}
$$

To show equality suppose $x \notin R_{i}$. If $x \in R^{\prime}$ then there exists $n \neq i$ such that $x \in R_{n}$ so that $x \notin D_{n}$. Thus if $x$ is not in the right set of the above equation it cannot be in the left set so that the equality obtains, which proves that $R_{i}$ is a convex polyhedron and hence a member of $\mathscr{R}_{0}$.

To see that $\mathscr{R}_{0}$ is a half ring which satisfies the finite chain condition in the event that $\mathscr{R}$ is such a half ring, suppose 


$$
\left\{R_{i} \mid i=1,2, \cdots, k ; R=R_{1}, R^{\prime}=R_{k}\right\}
$$

is the finite chain from $R$ to $R^{\prime}\left(R, R^{\prime} \in \mathscr{R}_{0}\right.$ and $\left.R \subset R^{\prime}\right)$. Let $i$ be arbitrarily chosen. Since the preceding paragraph implies $R_{i+1} \backslash R_{i} \in \mathscr{R}_{0}$ we need only show $R_{i} \in \mathscr{R}_{0}$. From Theorem 2.1, there exists a demispace $D_{n}$ such that $D_{n} \supset R_{n}$ and $X \backslash D_{n} \supset R_{n+1} \backslash R_{n}$ for all $n$. Thus reasoning similar to the above, it follows that

$$
R^{\prime} \cap\left[\bigcap_{n \geqq i} D_{n}\right]=R_{i}
$$

which completes the proof.

Let $\mathscr{N}$ be a translation invariant collection of convex sets which forms a neighborhood base for a linear topological space. $\mathscr{N}$ is said to be absorbing if $a N \in \mathscr{N}$ whenever $a>0, N \in \mathscr{N}$ and $N$ is a neighborhood of the origin.

COROLlaRY 4.3. If $\mathscr{R} \backslash\{\varnothing\}$ is an absorbing neighborhood base such that $\mathscr{R}$ is a semi-ring that satisfies the finite sequence condition, then each $R \in \mathscr{R}$ is a convex polyhedron.

Proof. If $R \in \mathscr{R}$ is a neighborhood of the origin then since $\mathscr{R} \backslash\{\varnothing\}$ is absorbing, $2 R \in \mathscr{R}$. Moreover, it easily follows that $\mathrm{cl}(R) \subset$ $\operatorname{int}(2 R)$. The reasoning presented in paragraph 1 of the preceding proof now applies to show that $R$ is a convex polyhedron and the assertion then follows from the translation invariance of $\mathscr{R}$.

The following theorem completes our characterization of the weak topology in terms of semi-rings of convex sets.

THEOREM 4.4. The weak topology of linear topological space always has an absorbing half ring of convex polyhedra which satisfies the finite chain condition as an equivalent neighborhood base.

Proof. Let $\Gamma$ be the collection of all continuous linear functionals and $\Gamma^{\prime}$ be a maximal subcollection of linearly independent members of $\Gamma$. Let $\mathscr{D}$ be the collection of all half spaces either of the form

$$
D_{r}^{+}=\{x \in X \mid f(x) \leqq c\}
$$

or

$$
D_{r}^{-}=\{x \in X \mid f(x)>c\}
$$

where $c$ ranges over all real numbers and $f$ over all $\Gamma^{\prime}$. By Theorem 3.1 , the collection $\mathscr{R}$ of all convex polyhedra $R$ which has a finite subset of $\mathscr{D}$ as a representative class is a half ring which satisfies the finite chain condition. Since $\Gamma^{\prime}$ is a linearly independent set, it 
easily follows that each nonvoid member of $\mathscr{R}$ has a nonempty interior. Thus $\mathscr{R} \backslash\{\varnothing\}$ is a neighborhood base for a linear topology which is no finer than the weak topology. But since each member of $\Gamma$ is continuous in the topology induced by $\mathscr{R} \backslash\{\varnothing\}$, this topology must be at least as fine as the weak topology.

We now turn our attention to characterizing the subspaces of the Banach space $\left(c_{0}\right)$ in terms of half rings.

THEOREM 4.5. If $X$ is a normed linear space, the following are equivalent:

1. $X$ is linearly homeomorphic to a subspace of $\left(c_{0}\right)$.

2. $X$ has an entire half ring $\mathscr{R}$ of convex polytopes with countably many faces such that $\mathscr{R} \backslash\{\varnothing\}$ is an absorbing neighborhood base.

3. $X$ contains a half ring $\mathscr{R}$ of convex sets such that $\mathscr{R} \backslash\{\varnothing\}$ neighborhood base.

Proof. To see that 1. implies 2., we define a convex subset $R$ of $\left(c_{0}\right)$ to be a half open rectangle if there exists a sequence $\left\{a_{i} \mid i=\right.$ $1,2, \cdots\}$ of positive extended real valued numbers and a sequence $\left\{b_{i} \mid i=1,2, \cdots\right\}$ of negative extended real valued numbers such that:

(a) $\operatorname{Lim} \inf _{i} a_{i}>0$,

(b) $\operatorname{Lim} \sup _{i} b_{i}<0$,

(c) $R=\left\{x \in\left(c_{0}\right) \mid b_{i}<x_{i} \leqq a_{i}\right\}$.

If $\mathscr{R}$ is the collection of all half open rectangles and the null set, then $\mathscr{R}$ can be seen to be an entire half ring of convex sets such that $\mathscr{R} \backslash\{0\}$ forms an absorbing neighborhood base for $\left(c_{0}\right)$. Now if $Y$ is a subspace of $\left(c_{0}\right)$ then it follows that the collection $\{R \cap Y \mid R \in \mathscr{R}\}$ is also an entire half ring of convex sets which without the null set forms an absorbing neighborhood base. That each of its members is a convex polytope with countably many faces, follows from Theorem 3.2. Thus every space $X$ which is linearly homeomorphic to a subspace of $\left(c_{0}\right)$ satisfies condition 2 .

Since 2 . obviously implies 3 . we need only prove that 3 . implies 1 . To accomplish this we can use the regularity of the topological space $X$ to prove the existence of a pair $R, R^{\prime}$ of bounded nondegenerate members of the half ring such that $\operatorname{cl}(R) \subset \operatorname{int}\left(R^{\prime}\right)$. Let $\left\{R_{i} \mid i=\right.$ $1,2, \cdots\}$ be an ascending chain from $R=R_{1}$ to $R^{\prime}=\bigcup_{i} R_{i}$. From Theorem 2.1, for each integer $i$, there exists a demi-space $D_{i}$ such that $D_{i} \supset R$ and $X \backslash D_{i} \supset R_{i}$. But then it is easily seen, by methods similar to those employed in the proof of Theorem 4.2, that $R$ is a convex polytope with $\left\{D_{i} \mid i=1,2, \cdots\right\}$ as a representative class of demi-spaces. Thus Theorem 2.3 and Corollary 2.4 imply that $\mathrm{cl}(R)$ is a bounded nondegenerate convex polytope (in the sense of [4] as well 
as in our sense) with a countable number of faces. Since we have already seen this to be a sufficient condition for $X$ to be linearly homeomorphic to a subspace of $\left(c_{0}\right)$ in [4], the proof is complete.

Corollary 4.6. A necessary and sufficient condition that a normed linear space $X$ be isomorphic to a subspace of $\left(c_{0}\right)$ is that it contains an entire half ring of convex sets which has at least one bounded nondegenerate member.

Proof. From Theorem 3.2 every member of the half ring must be a convex polytope with a countable number of faces. Thus it follows from Theorem 2.3 that the closure of each of its nondegenerate members is also such a convex polytope. The sufficiency of the condition now follows from the result of [4] mentioned above. The necessity is a consequence of the preceding theorem.

5. Measure theoretic properties of semi-rings. Zaanen [8] has shown that a countable additive non-negative set function defined on a semi-ring $R$ which satisfies the finite sequence property can be extended to such a function on the $\sigma$-ring $\sigma[\mathscr{R}]$ generated by $\mathscr{R}$. Here we will extend this result by removing the restriction that $\mathscr{R}$ satisfies the finite sequence property. Since a half ring is a semi-ring, note that this result is still valid in case $\mathscr{R}$ is a half ring. In the event that $\mathscr{R}$ is a half ring which satisfies the finite chain condition, von Neumann [6] has shown that finitely additive nonnegative set functions can be extended to the ring generated by $\mathscr{R}$. As seen by the following example this result is not valid for an arbitrary half ring.

Consider the half ring $\mathscr{R}$ defined in the proof of Theorem 4.5. If $R$ is an arbitrary member of this half ring as defined initially in the proof, let

$$
\omega(R)=\operatorname{Lim} \inf a_{i}-\operatorname{Lim} \sup b_{i} .
$$

To construct the counter example, we will consider the subclass $\mathscr{R}_{0}=\{R \in \mathscr{Z}: \omega(R)<\infty\}$. Since $\omega\left(R_{1}\right) \leqq \omega\left(R_{2}\right)$ whenever $R_{1} \subset R_{2}$ and $R_{1}, R_{2} \in \mathscr{R}$, it is clear that $\mathscr{R}_{0}$ is itself a half ring. If we define an extended real valued nonnegative set function $\mu$ by

$$
\mu(R)=\Pi_{i}\left[\left(a_{i}-b_{i}\right) / \omega(R)\right],
$$

then the definition of implies that $\mu$ is well defined. To see that $\mu$ is finitely additive, let $R_{1}$ and $R_{2}$ be two disjoint members of $\mathscr{R}_{0}$ such that $R_{1} \cup R_{\varepsilon}=R \in \mathscr{R}_{0}$. It follows that $R_{1}$ and $R_{2}$ differ from $R$ in exactly one coordinate, say the $j$ th. Thus

$$
R_{1}=\left\{x \in\left(c_{0}\right) \mid b_{i}<x_{i} \leqq a_{i}, i \neq j\right\} \cap\left\{x \in\left(c_{0}\right) \mid b_{j}^{1}<x_{j} \leqq a_{j}^{1}\right\}
$$


and

$$
R_{2}=\left\{x \in\left(c_{0}\right) \mid b_{i}<x_{i} \leqq a_{i}, i \neq j\right\} \cap\left\{x \in\left(c_{0}\right) \mid b_{j}^{2}<x_{j} \leqq a_{j}^{2}\right\} .
$$

But since $R_{1} \cup R_{2}=R$ and $R_{1} \cap R_{2}=\varnothing$ we may assume without loss of generality that

$$
b_{j}=b_{j}^{2}<a_{j}^{2}=b_{j}^{1}<a_{j}^{1}=a_{j} .
$$

Therefore

$$
\mu\left(R_{1}\right)+\mu\left(R_{2}\right)=\left(\frac{a_{j}^{1}-b_{j}^{1}}{R(\omega)}+\frac{a_{j}^{2}-b_{j}^{2}}{R(\omega)}\right)\left(\prod_{i \neq j}\left[\frac{a_{i}-b_{i}}{R(\omega)}\right]\right)=\mu(R) .
$$

That $\mu$ has no finitely additive extension to the ring generated by $\mathscr{R}_{0}$ follows if we let

$$
R^{\prime}=\left\{x \in\left(c_{0}\right) \mid-2<x_{i} \leqq 2\right\}
$$

and

$$
R^{\prime \prime}=\left\{x \in\left(c_{0}\right) \mid-1<x_{i} \leqq 1 \text { for } i \neq 1\right\} \cap\left\{x \in\left(c_{0}\right) \mid-2<x_{1} \leqq 2\right\} .
$$

Then $R^{\prime \prime} \subset R^{\prime}$ while $\mu\left(R^{\prime \prime}\right)=2>1=\mu\left(R^{\prime}\right)$.

Let $\mathscr{R}$ be a semi-ring which contains $\varnothing$ and let $\mathscr{S}$ be the collection of all sets which can be expressed as the union of a countable number of pairwise disjoint members of $\mathscr{R}$. Let $\mathscr{C}$ be the set of all $M \in \mathscr{S}$ for which there exists $M^{\prime} \in \mathscr{S}$ disjoint from $\mathscr{C}$ such that $M \cup M^{\prime} \in \mathscr{R}$. Note that since $\varnothing \in \mathscr{R}$, there is no question of the existence of $\mathscr{C}$ and that $\mathscr{C}$ always contains $\mathscr{R}$. Observe further that $M^{\prime}$ is not necessarily unique for a given $M$ and the notation $M^{\prime}$ will be used to specify an arbitrary such member of $\mathscr{l}$.

THEOREM 5.1. Il is a half ring which satisfies the finite chain condition. In fact, $M \backslash N \in \mathbb{C l}$ whenever $M, N \in \mathscr{L}$.

Proof. (a). Let $M, N \in \mathscr{C l}$. Since

$$
(M \cap N) \cup\left[\left(M^{\prime} \cap N^{\prime}\right) \cup\left(M^{\prime} \cap N\right) \cup\left(M \cap N^{\prime}\right)\right]=\left(M \cup M^{\prime}\right) \cap\left(N \cup N^{\prime}\right) \in \mathscr{R}
$$

and since the bracketed part of the above equation can be expressed as the union of countably many pairwise disjoint members of $\mathscr{R}$ which are each also disjoint from $M \cap N$, it follows that $M \cap N \in \mathscr{l}$. Moreover $(M \cap N)^{\prime}$ can be selected to be $\left(M^{\prime} \cap N^{\prime}\right) \cup\left(M^{\prime} \cap N\right) \cup\left(M \cap N^{\prime}\right)$.

(b). If $R \in \mathscr{R}, N \in \mathscr{L}$, then $R \backslash N \in \mathscr{l}$. For by (a), $N \cap R$ can be expressed as the union of countably many pairwise disjoint members of $\mathscr{R}$. Since $N \cap R$ is disjoint from $R \backslash N$ and $(R \backslash N) \cup(N \cap R)=R$ we need only show that $R \backslash N$ can be expressed as the union of a 
countable number of pairwise disjoint members of $\mathscr{R}$. This is probably best realized by expressing $R \backslash N$ as

$$
R \backslash N=\left[R \backslash\left(N \cup N^{\prime}\right)\right] \cup\left[N^{\prime} \cap R\right]
$$

and pointing out that the bracketed expressions are disjoint and can be expressed as a countable union of pairwise disjoint members of $\mathscr{R}$.

(c). To show that $M \backslash N \in \mathscr{C l}$ for an arbitrary pair $M, N \in \mathscr{C}$; let $\left\{R_{i}(M)\right\}$ be a countable subcollection of pairwise disjoint members of $\mathscr{R}$ whose union is $M$. Then

$$
M \backslash N=\bigcup_{i}\left[R_{i}(M) \backslash N\right] .
$$

From (b), each $R_{i}(M) \backslash N$ can be expressed as the union of countably many pairwise disjoint members of $\mathscr{R}$. But since the collection $\left\{R_{i}(M) \backslash N\right\}$ is itself pairwise disjoint, we need only find a suitable $(M \backslash N)^{\prime}$. But since $(M \backslash N) \cup\left[(M \cap N) \cup M^{\prime}\right]=M \cup M^{\prime} \in \mathscr{R}$, we note that such a set is the bracketed part of the above equation and the proof is complete.

It is easily seen that $\mathscr{C l}$ is not a ring if one selects $\mathscr{R}$ to be the collection of all right open and left closed intervals on the real line whose Lebesgue measure is no greater than unity. This same example also shows that the ring generated by . /l is not necessarily that generated by $\mathscr{R}$. The $\sigma$-ring generated by $\mathscr{R}$ and $\mathscr{l l}$ are of course synonymous.

THEOREM 5.2. Every countably additive extended real valued nonnegative set function $\mu_{1}$ defined on a semi-ring $\mathscr{R}$ has a unique nonnegative countably additive extension to the half ring $\mathscr{A}$.

Proof. If $M \in \mathscr{C l}$, let $\left\{R_{1 i}\right\}$ be a countable collection of pairwise disjoint members of $\mathscr{R}$ whose union is $M$. Since the only possible countably additive extension $\ell_{1}$ to $\mathscr{l}$ must satisfy

$$
\mu_{1}(M)=\Sigma_{i} \mu_{0}\left(R_{1 i}\right)
$$

we take this to be the definition of $\mu_{1}$. In order to show that $\mu_{1}$ is well defined on $\mathscr{C l}$, let $\mathscr{C}=\bigcup_{j} R_{2 j}$ where $\left\{R_{2 j}\right\}$ is a countable subcollection of pairwise disjoint members of $R$. Then

$$
\begin{aligned}
\Sigma_{i} \ell_{0}\left(R_{1 i}\right) & =\Sigma_{i} \Sigma_{j} \mu_{0}\left(R_{1 i} \cap R_{2 j}\right) \\
& =\Sigma_{j} \Sigma_{i} \mu_{0}\left(R_{1 i} \cap R_{2 j}\right) \\
& =\Sigma_{j} \mu_{0}\left(R_{2 j}\right) .
\end{aligned}
$$

Thus $\mu_{1}$ is well defined.

To see that $\mu_{1}$ is countably additive on $\mathscr{l l}$, let $\left\{M_{i}\right\}$ be a counta- 
ble collection of disjoint members of $M$. For each $i$, let $\left\{R_{i j}\right\}$ be a countable subcollection of disjoint members of th whose union is $M_{i}$. Then

$$
\begin{aligned}
\Sigma \mu_{1}\left(M_{i}\right) & =\Sigma_{i} \Sigma_{j} \mu_{0}\left(R_{i j}\right) \\
& =\Sigma_{i, j} \mu_{0}\left(R_{i j}\right) \\
& =\mu_{1}\left(\bigcup_{i} M_{i}\right)
\end{aligned}
$$

(where the notation $\Sigma_{i, j} \mu_{0}\left(R_{i j}\right)$ is used to signify that the real numbers $\left\{\mu_{0}\left(R_{i j}\right)\right\}$ are summed under any ordering which eventually counts all of the terms exactly once; it is readily seen that the limit of the given series is independent of all such summation orders).

If $\mu_{0}$ is a countably additive nonnegative set function which is $\sigma$-finite on a semi-ring $\mathscr{R}$ (i.e. for each $R \in \mathscr{R}$ there is a countable collection $\left\{R_{i}\right\} \subset \mathscr{R}$ such that $\mu_{0}\left(R_{i}\right)<\infty$ for each $i$ and $\left.\bigcup_{i} R_{i} \supset R\right)$ then clearly its extension $\mu_{1}$ is $\sigma$-finite on $\mathscr{L}$. But from [6, Th. 10.1.12 and Th. 10.1.14], $\mu_{1}$ (without $\sigma$-finiteness) can be extended uniquely to the ring $R[\mathbb{C}]$ generated by $M$. If we denote this extension by $\mu_{2}$ then the form of $R[-/ C \mid[6$, Th. 10.1.2], indicates that $\mu_{2}$ is $\sigma$-finite. Thus it follows from [2, Chap. 3, Sec. 13, Th. A] that:

COROLLARY 5.3. Every nonnegative countably additive set function $\mu_{0}$ on a semi-ring $\mathscr{R}$ has a nonnegative countably additive extension to the $\sigma$-ring, $\sigma[\mathscr{R}]$ generated by $\mathscr{R}$. If, moreover, $\mu_{0}$ is $\sigma$-finite on $\mathscr{R}$, then its extension is unique.

The author is indebted to Professor R. E. Fullerton for his interest and assistance, and Professor P. C. Hammer for introducing and discussing the notion of a demi-space with him.

\section{REFERENCES}

1. N. Dunford and J. Schwartz, Linear Operators (I), New York, 1958.

2. P. R. Halmos, Measure Theory, New York, 1950.

3. P. C. Hammer, Maximal convex sets, Duke Math. J. 22 (1955), 103-106.

4. P. H. Maserick, Convex polytopes in linear spaces, To appear in the Illinois J. Math.

5. - The structure of translation half rings in linear spaces, Proc. Amer. Math. Soc. 10 (1959), 133-139.

6. J. von Neumann, Functional Operators (I), Princeton, 1950.

7. H. Weyl, The elementary theory of convex polyhedra, Annals of Mathematics Studies, 24 (1950), 3-18.

8. A. C. Zaanen, Linear Analysis, Amsterdam, 1956.

UNIVERSITY OF MARYLAND

AND

The Pennsylvania State University 


\section{PACIFIC JOURNAL OF MATHEMATICS}

\section{EDITORS}

\section{H. SAMELSON}

Stanford University

Stanford, California

R. M. Blumenthal

University of Washington

Seattle, Washington 98105

\author{
*J. DugundJI \\ University of Southern California \\ Los Angeles, California 90007 \\ RICHARD ARENS \\ University of California \\ Los Angeles, California 90024
}

\section{ASSOCIATE EDITORS}
E. F. BECKENBACH
B. H. NeUManN
F. WolF
K. YosIDA

\section{SUPPORTING INSTITUTIONS}

UNIVERSITY OF BRITISH COLUMBIA
CALIFORNIA INSTITUTE OF TECHNOLOGY
UNIVERSITY OF CALIFORNIA
MONTANA STATE UNIVERSITY
UNIVERSITY OF NEVADA
NEW MEXICO STATE UNIVERSITY
OREGON STATE UNIVERSITY
UNIVERSITY OF OREGON
OSAKA UNIVERSITY
UNIVERSITY OF SOUTHERN CALIFORNIA

UNIVERSITY OF BRITISH COLUMBIA

UNIVERSITY OF CALIFORNIA

MONTANA STATE UNIVERSITY

NEW MEXICO STATE UNIVERSITY

OREGON STATE UNIVERSITY

OSAKA UNIVERSITY

UNIVERSITY OF SOUTHERN CALIFORNIA

\author{
STANFORD UNIVERSITY \\ UNIVERSITY OF TOKYO \\ UNIVERSITY OF UTAH \\ WASHINGTON STATE UNIVERSITY \\ UNIVERSITY OF WASHINGTON \\ AMERICAN MATHEMATICAL SOCIETY \\ CHEVRON RESEARCH CORPORATION \\ TRW SYSTEMS \\ NAVAL ORDNANCE TEST STATION
}




\section{Pacific Journal of Mathematics \\ Vol. 17, No. $1 \quad$ January, 1966}

Carlos Jorge Do Rego Borges, On stratifiable spaces ................ 1

Felix Earl Browder, Topological methods for non-linear elliptic equations of

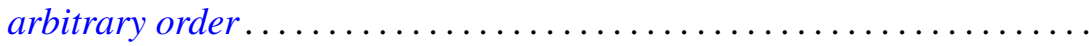

Gustave Choquet, Harry Corson and Victor Klee, Exposed points of convex

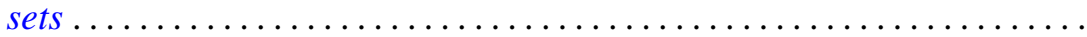

Phillip Emig, Remarks on the defect sum for a function meromorphic on an open Riemann surface ................................ 45

Ruth Goodman, A certain class of polynomials .................. 57

Sidney (Denny) L. Gulick, The bidual of a locally multiplicatively-convex

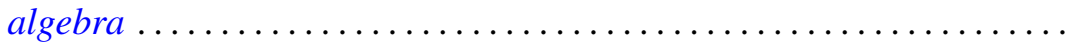

Eugene Carlyle Johnsen, Integral solutions to the incidence equation for finite projective plane cases of orders $n \equiv=2(\bmod 4) \ldots \ldots \ldots \ldots . .67$

Charles N. Kellogg, Centralizers and $H^{*}$-algebras .................. 121

Michael Lodato, On topologically induced generalized proximity relations. II .......................................... 131

P. H. Maserick, Half rings in linear spaces ..................... 137

Kathleen B O'Keefe, On a problem of J. F. Ritt .................... 149

Galen Lathrop Seever, Nonnegative projections on $C_{0}(X) \ldots \ldots \ldots \ldots$

Lawrence A. Shepp, Gaussian measures in function space ............ 167

Robert Charles Thompson, Classes of definite group matrices ........... 175 